\title{
The Online Authenticity Paradox: What Being "Authentic" on Social Media Means, and Barriers to Achieving It
}

\author{
OLIVER L. HAIMSON, University of Michigan, USA \\ TIANXIAO LIU, University of Michigan, USA \\ BEN ZEFENG ZHANG, University of Michigan, USA \\ SHANLEY CORVITE, University of Michigan, USA
}

People often strive to present themselves authentically on social media, but this may not be possible for everyone. To understand how people view online authenticity, how it relates to social media sharing behaviors, and whether it is achievable, we interviewed 28 social media users who had recently experienced major life transitions. We found that to many participants, online authenticity required presenting a consistent, positive, and "true" self across online and offline contexts. Though most stated that they considered online authenticity achievable, their social media self-disclosure behaviors around life transitions revealed what we call the online authenticity paradox: people strive to achieve online authenticity, yet because doing so requires sharing negative experiences on social media, online authenticity is often unreachable, or is possible only at great personal cost - especially for those with marginalized identities and difficult life experiences.

CCS Concepts: • Human-centered computing $\rightarrow$ Empirical studies in HCI; Empirical studies in collaborative and social computing.

Additional Key Words and Phrases: online authenticity; social media; self-disclosure; online sharing behaviors; life transitions

\section{ACM Reference Format:}

Oliver L. Haimson, Tianxiao Liu, Ben Zefeng Zhang, and Shanley Corvite. 2021. The Online Authenticity Paradox: What Being "Authentic" on Social Media Means, and Barriers to Achieving It. Proc. ACM Hum.-Comput. Interact. 5, CSCW2, Article 423 (October 2021), 18 pages. https://doi.org/10.1145/3479567

\section{INTRODUCTION}

Most people strive to present themselves in ways they consider authentic, in both online and physical world settings [71]. However, what authenticity means is highly subjective and socially constructed $[32,56]$. People often struggle with online self-presentation when aspects of their identities and experiences are marginalized, stigmatized, or otherwise not easily shareable $[1,6,38]$. While it is clear that online authenticity can be complex and elusive [55], particularly for people with marginalized identities or difficult experiences [15], open questions remain about social media users' conceptions of online authenticity and its relationship to their online self-presentation in the

Authors' addresses: Oliver L. Haimson, haimson@umich.edu, University of Michigan, USA; Tianxiao Liu, tialiu@umich.edu, University of Michigan, USA; Ben Zefeng Zhang, bzfzhang@umich.edu, University of Michigan, USA; Shanley Corvite, scorvite@umich.edu, University of Michigan, USA.

Permission to make digital or hard copies of all or part of this work for personal or classroom use is granted without fee provided that copies are not made or distributed for profit or commercial advantage and that copies bear this notice and the full citation on the first page. Copyrights for components of this work owned by others than the author(s) must be honored. Abstracting with credit is permitted. To copy otherwise, or republish, to post on servers or to redistribute to lists, requires prior specific permission and/or a fee. Request permissions from permissions@acm.org.

(c) 2021 Copyright held by the owner/author(s). Publication rights licensed to ACM.

2573-0142/2021/10-ART423 \$15.00

https://doi.org/10.1145/3479567

Proc. ACM Hum.-Comput. Interact., Vol. 5, No. CSCW2, Article 423. Publication date: October 2021. 
context of major life events and transitions ${ }^{1}$. In line with Anderson et al. [5], in this work major life transitions refer to life changes that people consider to have a major impact on them. Such life transitions include both prevalent life events (e.g., graduation from college, starting a new job) and events that are less common but require major readjustment (e.g., death of a loved one, recovery from addiction).

In this study, we addressed the following research questions:

RQ1: How do people who have recently experienced life transitions view online authenticity?

RQ2: Do these people consider online authenticity to be achievable?

RQ3: How does people's conception of online authenticity relate to their self-disclosure and selfpresentation on social media during major life transitions?

To answer these research questions, we conducted interviews $(n=28)$ with people who had experienced major life transitions within the past two years to investigate social media selfdisclosure behaviors related to both positive and negative life experiences.

We contribute an empirical understanding of how people consider online authenticity, and its relation to their online sharing behaviors during life transitions. Our qualitative analysis revealed that for many, being authentic meant presenting a "true" and consistent self across online and offline contexts, which necessitated sharing both positive and negative content on social media. Yet, though sharing negative content online was difficult and many chose not to (often in anticipation of negative reactions from audiences), most stated that they believed online authenticity was achievable, both for themselves and for others. We contribute a concept that we call the online authenticity paradox: people strive to achieve online authenticity, yet because doing so requires sharing negative or sensitive experiences with broad audiences, it is out of reach for many especially people with marginalized identities and difficult or stigmatized life experiences.

\section{RELATED WORK}

\subsection{Sharing Difficult/Sensitive Content Online}

Research has shown that when people face difficult experiences or their lives are disrupted by major transitions, their social media behaviors shift [35, 65]. People often want to share challenges and emotions with others [61], and many turn to social media to disclose difficult information and gain social support [2]. We describe a large body of previous research about how people consider audiences and platforms when they choose whether to share difficult and sensitive content online, to situate and motivate our study that specifically examined online authenticity's relationship to self-presentation and self-disclosure on social media.

Literature on online presentation has examined how people share difficult content, including but not limited to death of a loved one [10], job loss [11], and relationship breakups [36]. A growing body of research has examined social media use in sharing negatively perceived and stigmatized events, such as pregnancy loss [2], sexual abuse [3], and addiction [13]. Sharing difficult content can lead to positive outcomes such as resources and support $[1,14]$, and negative outcomes such as rejection and further stigmatization [8]. Because sharing negative events can be difficult due to factors such as privacy and stigma, people often choose not to disclose them on social media [1]. For people with stigmatized identity facets, disclosing identity aspects is complex; thus, people often conceal stigmatized identities [30]. One of the most common social media disclosure behaviors is not to disclose $[1,36]$. However, seeing others receive support after sharing similar experiences

\footnotetext{
${ }^{1}$ Hereafter referred to simply as "life events" or "life transitions" rather than "life events and transitions." Some life transitions involve multiple stages and take long periods of time to complete (e.g., divorce, gender transition), while others take place on a particular day yet also involve longer identity change processes (e.g., pregnancy, job loss). We use "life transitions" as an umbrella term to encompass life experiences involving both moments and processes of change.
}

Proc. ACM Hum.-Comput. Interact., Vol. 5, No. CSCW2, Article 423. Publication date: October 2021. 
online can influence individuals to share as well [1]. While each of these studies examined people's online sharing behaviors around particular difficult or stigmatized life experiences, our study details how people's conceptions of authenticity may relate to a wide range of life transition experiences.

Online audiences greatly impact people's self-presentation and decisions whether and how to share difficult or sensitive content online. Context collapse occurs when multiple incongruent audiences are present in the same digital space [55]. Many online spaces include people from different facets of one's life, which can make sharing decisions difficult [55]. To manage context collapse, social media users often create boundaries between separate audiences [23, 55, 68]. This separation can be accomplished via multiple accounts on one platform [68], each of which is tailored for a particular imagined audience [55], or by using privacy affordances to segment content to only some parts of a large, diverse network [73], or by participating in communities in separate online spaces $[35,51]$. Especially in health contexts, people with similar conditions or diagnoses tend to gather in online spaces where they can share with, form connections with, and receive support from similar others [24, 25, 47, 51, 52, 67, 80]. Additionally, LGBTQ+ people often manage context collapse by tailoring their identity presentations to particular audiences, such as those to whom they have and have not disclosed their LGBTQ+ identity, and by maintaining separate online audiences for these different identity performances [20, 23].

Social media norms, which typically emphasize positive self-presentations $[72,76,77]$, can also influence online disclosure. Such factors include positivity bias and social desirability bias. "Positivity bias" refers to people's preference for positive information [42]. Online, this means generally favoring "positive forms of authenticity over negative ones" [59]. It encourages sharing positive experiences over difficult content, despite the latter's crucial role in the relationship between well-being and authentic self-presentation [59]. Social desirability bias - "the tendency... to deny socially undesirable traits or qualities and to admit to socially desirable ones" [57] - encourages showcasing positive social selves [70], hindering exhibition of self-aspects related to negatively perceived and stigmatized events.

As sharing both positive and negative content on social media may be considered "authentic" [59], we next discuss authenticity and how it has been applied to online sharing. To our knowledge, few studies have explored sharing difficult content across a variety of contexts and experiences, and particularly in relation to online authenticity; our study addresses this gap.

\subsection{Authenticity}

Authenticity is complex, highly subjective, and socially constructed [56]. The term usually carries positive connotations, and is considered a "basic need" that people strive to fulfill [71]. Maintaining authenticity requires effort, as it is not only internal, but also requires "allowing the outside world access to one's inner self" [6]. Some have argued that authenticity is artificial and an "idealized representation of reality" [32] that is performed rather than inherent [56]. Thus, authenticity must be critically examined, as we do in this work.

People often strive to be authentic both in virtual and physical settings, but online authenticity can be ambiguous $[55,63]$ due to complex relationships between one's "real, embodied self" and "virtual persona" [21]. Rather than being fixed, identity is flexible, changeable, and highly dependent upon context [54]. Research has shown that people are more able to express their "true self" in online settings where they can be anonymous [7]. We further examine online authenticity's complex relationship to online self-presentation in different contexts.

Authenticity online is closely linked with self-presentation and self-disclosure on social media [64]. Though some have argued that authentic identity online is constructed rather than internal [31], social media sites often encourage presenting a single identity or combining potentially disparate facets into one identity [16]. Such requirements reduce user agency by assuming a 
"normative, decontextualized concept of self" that is "partly constructed by the application, not the user" [53]. boyd [9] argued that all social media profiles are performances where people give off certain impressions; thus, those who present "fake" versions of themselves are no less authentic than others.

People often struggle with online self-presentation when aspects of their identities are marginalized, stigmatized, or otherwise not easily shareable $[1,6,38,58]$. For instance, Facebook demands authentic identity presentation (e.g., by enforcing "real name" policies) which can be difficult for some marginalized users, such as transgender people who may not have identification that matches their name, or abuse survivors who use pseudonyms due to safety concerns [38]. Having multiple, sometimes incongruent, identity aspects can lead to self-doubt [26]. Exhibiting oneself inauthentically may reduce both social connections and well-being [33, 59]. Thus, people employ strategies such as creating "finstas," ("fake" Instagram accounts) [22, 70, 79] and anonymity [3] to present authentic selves online. Some may experience what Gergen [26] called pastiche personality - the acceptance that authenticity is unachievable, which enables living with multiple identities without guilt.

Researchers have attempted to measure authenticity using quantitative surveys and validated scales [28, 39, 48, 50, 78]. Wood et al.'s highly-cited Authenticity Scale [78] is meant to measure "a clear definition of the construct" of authenticity made up of three factors: self-alienation, authentic living, and accepting external influence. Authenticity is also sometimes measured as the (lack of) difference between ideal and actual self $[39,59]$. Many works have shown positive correlations between authenticity measures and well-being $[33,50,59,78]$. Thus, researchers have considered ways to make online authenticity more achievable, such as via privacy settings [71]. In this work, we consider online authenticity, its achievability, and implications for social media platforms in depth.

Prior work in branding and social media has paid specific analytical attention to the concept of authenticity [6, 18, 40]. For example, Banet-Weiser argued authenticity should not be only "understood and experienced as the pure, inner self of the individual, it is also a relationship between individuals and commodity culture" [6]. In Banet-Weiser's [6] view, online authenticity is both outside of and yet entwined with consumer brand cultures. However, this authenticity-related literature is centered around branding and social media; less is known about social media users' conceptions of authenticity and how they relate to online self-presentation in the context of life transitions. We expand on previous work by examining perceptions of what it means to be authentic online across different life events and experiences, and examine online authenticity in the context of life transitions and self-disclosures.

\section{METHODS}

We conducted semi-structured interviews $(n=28)$ to understand perceptions of authenticity online and how they correspond to social media sharing. Our inclusion criteria required that participants: 1) used social media; 2) had gone through major life transitions during the past two years; 3 ) had disclosed one or more of these on social media; and 4) were 18 or older. We specified a two-year timespan because a longer timespan would cause recall difficulties [44], while a shorter one would leave out many experiences. The study was approved by our institution's ethics review board.

We recruited 17 participants by sharing the study information and a link to our screening survey on our personal social media accounts, which was widely disseminated beyond our personal networks, and 11 more via User Interviews, a research recruiting platform. Our screening survey enabled us to choose a diverse sample based on demographics, life experiences, and social media sharing behaviors. Participant demographics and social media platforms used are reported in Table 1. All reported more than two major life events during the past two years, in categories

Proc. ACM Hum.-Comput. Interact., Vol. 5, No. CSCW2, Article 423. Publication date: October 2021. 
Table 1. Participant demographics and social media platforms used.

\begin{tabular}{lr}
\hline & $\begin{array}{r}\text { count } \\
\text { (percentage) } \\
(n=28)\end{array}$ \\
\hline Gender & $13(46.4 \%)$ \\
Woman & $14(50.0 \%)$ \\
Man & $2(7.1 \%)$ \\
Nonbinary & $2(7.1 \%)$ \\
Transgender (lower bound) & \\
\hline Race / Ethnicity & $1(3.6 \%)$ \\
American Indian or Alaska Native & $5(17.9 \%)$ \\
Asian & $4(14.3 \%)$ \\
Black / African American & $4(14.3 \%)$ \\
Hispanic / Latine & $14(50.0 \%)$ \\
White & $1(3.6 \%)$ \\
Mixed & \\
\hline Age & $8(28.6 \%)$ \\
18-24 & $13(46.4 \%)$ \\
$25-34$ & $2(7.1 \%)$ \\
35-44 & $2(7.1 \%)$ \\
$45-54$ & $1(3.6 \%)$ \\
$55-64$ & $2(7.1 \%)$ \\
65+ & \\
\hline Social media platforms used & $26(92.9 \%)$ \\
Facebook & $24(85.7 \%)$ \\
Instagram & $22(78.6 \%)$ \\
YouTube & $21(75.0 \%)$ \\
LinkedIn & $16(57.1 \%)$ \\
Twitter & $14(50.0 \%)$ \\
Reddit & $14(50.0 \%)$ \\
Snapchat & $7(28.6 \%)$ \\
TikTok & $7(25.0 \%)$ \\
WhatsApp & $7(25.0 \%)$ \\
Pinterest & $6(21.4 \%)$ \\
Discord & $4(14.3 \%)$ \\
\hline Pumblr & \\
Participants could choose multiple gender, \\
so percentages add up to greater than \\
\hline & $100 \%$. \\
\hline & \\
\hline
\end{tabular}

including relocation, relationships, career, education, health, death, and identity (see Table refTable 2). Each received a $\$ 30$ gift card.

Interviews, conducted between February and May 2020, averaged 59 minutes. Interviews were semi-structured to allow the topics most salient for participants. We asked about life transitions broadly, social media use broadly, impacts of social media on life and identity, sharing behaviors related to each transition experienced, and perceptions of authenticity on social media. Out of 28 participants, only 20 were asked directly about online authenticity. We conducted analysis on a related survey (not covered in this paper) concurrently with interviews, and authenticity's relationship with online sharing behaviors emerged as an important survey result. Additionally, a common theme that emerged from the first eight interviews was that people curate their posts 
Table 2. Summary of life transitions participants experienced.

\begin{tabular}{ll}
\hline Transition categories* & Specific life transitions participants experienced \\
\hline Health & $\begin{array}{l}\text { serious physical illness diagnosis, mental health struggles, serious in- } \\
\text { jury, accident, or physical ailment, major surgery, pregnancy, pregnancy } \\
\text { loss, recovery from addiction, began heavily using drugs or alcohol }\end{array}$ \\
\hline Financial & home purchase \\
\hline Relocation & $\begin{array}{l}\text { lost home/became homeless, move to different town/city within same } \\
\text { state, move within same town/city, move to a different state, move to a } \\
\text { different country, family member moved into/out of household }\end{array}$ \\
\hline Legal & went to jail or prison, released from jail or prison, lawsuit or legal action \\
\hline Relationships & marriage, engagement, divorce, ended/began serious romantic relation- \\
& ship \\
\hline Family relationships & new pet, gave birth/became a parent \\
\hline Death & death of loved one, death of parent, death of extended family member, \\
death of pet
\end{tabular}

and present themselves differently on different social media sites, conforming to each site's social norms and often leaving desirable yet inconsistent impressions to different social media networks. To deepen our understanding of this impression management pattern, which some participants described as related to their feelings of authenticity, we began asking interview participants about online authenticity directly after already conducting 8 interviews. We included all 28 interviews in data analysis for this paper because each informed the paper's themes. All were conducted via video chat, audio-recorded, and transcribed for analysis, with participants' consent.

We analyzed interview transcripts using open-coding and axial coding [17]. First, the research team each separately conducted line-by-line analysis of the first five interviews, allowing codes and themes to emerge from the data. We then discussed and refined codes and grouped them into larger themes. Three authors coded the remaining interviews, iteratively grouping data into new and existing codes and themes, and collaboratively refining the codebook. The research team met twice weekly throughout to discuss codes, themes, and connections between them. We organize the Results section based on three prominent themes in the data: what being authentic online means, whether it is considered achievable, and sharing behaviors surrounding positive and negative life events related to online authenticity.

\section{RESULTS}

\subsection{What does it mean to be authentic online?}

Interviews provided an in-depth understanding of how people think about authentic identity presentation on social media. Many participants stated or implied that they considered it important to be authentic online. Participants acknowledged that what this means differs for each person. In the words of P15, "I think that it... might depend on who you are as a person." P19 stated that "each individual is authentic unto themselves. I can define it for myself. You would probably have your

Proc. ACM Hum.-Comput. Interact., Vol. 5, No. CSCW2, Article 423. Publication date: October 2021. 
own definition.” As these quotes demonstrate, the concept is highly subjective. However, the most common ways participants described online authenticity were: presenting identity consistently across online and offline contexts, and presenting both positive and negative content online.

Many associated authenticity with presenting a "true" identity consistently across online platforms and offline contexts. For P13, authenticity meant "having your own style... sticking to the style, and not doing things that are unnatural." P22 said, "I think that's the most authentic you could be, is staying consistent through how you present yourself in person face to face, on a phone call, email, and also social media." Being authentic and presenting a "true" identity online involved telling the truth and being honest, being consistent, curating one's online content as little as possible, and interacting with others genuinely.

The most common description involved sharing both positive and negative content online, even though sharing negative content was more difficult. P27 said,

When I think about authenticity, I think about vulnerability, and sharing, and not creating something that is like a persona for social media... so many people only share the positives of their lives, but share those negatives!....I post pictures of my engagement, or being on a trip, but I don't post the photos of when I was in so much pain and couldn't get out of bed...

P27 encourages people to share both positive and negative life experiences ("share those negatives!"). However, describing her own posting behaviors, she mentions sharing positive, but not negative, experiences. Authenticity, to some, meant that in addition to sharing content that portrayed a positive self, one must share their views even when controversial, and also share more mundane content. P16 stated that

to really present yourself authentically, you'd have to document every single thing that's going on in your life... the exciting parts, the really sad parts and the day-to-day things too. Fust like, if you're going to get food, or if you're like cleaning up around the house...

Online authenticity involved presenting an online identity that mirrored participants' "true" identity and experiences, incorporating the positive, negative, exciting, and mundane.

Participants frequently voiced negative opinions about those in their networks whom they did not consider to present authentic identity online. P9 said, "I don't believe in lying or making things sound better than they are on the internet. I think that that's pretty irritating when people are just presenting a different identity that's probably not true to them." Some described particularly disliking those whom they thought bowed to pressure to present a certain image, or seek acceptance: "I feel like a lot of people try to be someone they're not or someone that other people want them to be, I guess to feel accepted or to try to fit in..." (P12). Statements like these demonstrate how some participants seemed to hold people in their networks to potentially unrealistic standards. That is, they described an expectation that people in their network should present themselves authentically, when (as we describe in the remainder of this paper) online authenticity may not actually be achievable, particularly for people experiencing life transitions or with marginalized identities.

Other participants described a more complex orientation in which one's online presentation differed depending on the platform, network, and audience. P20 said,

So if I'm talking with my close friends on WhatsApp, I'm definitely more of my real self. I think everybody does that....I don't think anybody's going on LinkedIn and talking about their breakfast. You're trying to show that you're professional...

Some described believing that a person could have multiple authentic versions of self online. In P9's words, 
I think for me, LinkedIn at work is an authentic version of myself and how I present myself at work. But when I direct message somebody on Instagram or have a conversation... that to me is this layer of personal identity that I choose to only share with certain people.

Though P24 sometimes withheld aspects of his identity online, he still considered his presentation authentic:

\section{I feel like I'm authentically representing my identity. But I also know that I'm withholding some parts of it. So I'm not lying by being what I am online, but also I'm not saying everything.}

Relatedly, P14 stated that despite using pseudonyms on some platforms, his presentation was authentic because his ideas and experiences were real. These examples show that for some, online authenticity can involve inconsistencies and differing presentations depending on context.

\subsection{Is online authenticity achievable?}

Given participants' conceptions of authenticity, some of which seemed difficult or unrealistic to implement, we asked whether being authentic online was achievable, for themselves and others. A majority ( $n=12$ out of 20 who were asked this question) reported that they thought it was achievable. For some it was simple: "Oh, yes, definitely achievable" (P25) or "Most definitely... for anyone" (P12). Others gave more complex answers, such as P27: "I don't think it's something that maybe comes naturally to some people... We like to see positive things. But I think if you find that balance, you could achieve that." Responses like these reflected views that online authenticity was achievable, but acknowledged potential difficulties.

A smaller group ( $n=4$ out of 20 ) considered online authenticity to be unachievable, such as P17:

I think it's unrealistic... social media involves... people from different social classes: high class, low class, middle class, it doesn't matter... So, I think that when people see that... other people are at a better place than they are, that kind of causes shame... And people start making posts about things that are not true or, you know, things that look a certain way but really aren't, in order to get that confidence back... that's always going to go on.

P17's response highlights how social comparison [75] can lead to heavily curated content, hindering authentic self-presentation.

Others ( $n=4$ out of 20$)$ saw online authenticity as only partially achievable. P23 said, "People don't want to put themselves in vulnerable positions...So it's not easy... we all go through the same things, and so I don't feel the need to pretend... I think it's achievable, but it won't be." This quote notes that because sharing vulnerable content is difficult, many will choose not to. Though P23 describes life experiences as somewhat universal ("we all go through the same things"), some face unique experiences that they would not be comfortable sharing with those who may not empathize or understand [1].

\subsection{Sharing behaviors of positive and negative experiences on social media}

Participants experienced a range of life transitions and events which we summarize in Table 2. Some life events were positive experiences for participants (e.g., marriage and home purchase), some were negative (e.g., becoming homeless, loss of loved one), and others were more complex processes that involved both positive and negative aspects (e.g., coming out as LGBTQ+, moving to a different state or country). Life events that involved negative emotions and stigma tended to involve more complicated decisions around whether or not to share about the event online, and which audiences to share with, supporting results from previous work (e.g., [2, 37, 60]). By asking what life events participants had shared or not online and why, we examined their online self-presentation and motivations for sharing. Participants revealed that with broad social media 
audiences, most tend to share positive events and not negative content. Such behavior contradicts participants' common definition of online authenticity, which required sharing both positive and negative content. Furthermore, we found that participants made selective disclosures to leave positive impressions, which is inconsistent with another requirement for online authenticity they described: curating content as little as possible. Some had shared negative life events online in the past, but after receiving negative reactions, were demotivated from sharing such content again.

Most avoided sharing negative content with broad online audiences, primarily posting about positive events. They typically shared milestones like weddings, graduation, babies, moving, and new jobs. Participants reported, "I shared... things that were worth announcements, so my wedding, my honeymoon, trips we go on..." (P13), and "[We shared] what happened when our daughter was born. We posted 'here's our daughter"' (P18). Participants also observed mostly positive disclosures in their online networks. P7 reflected,

A lot of people only post about the good life transitions, like moving to a new city, getting a brand new job, going to college... So everyone only sees the good stuff... I think that's just universal in every single social media site I've ever been on.

As demonstrated by these quotes, interviewees and their networks overwhelmingly posted positive content.

At the same time, many avoided posting about negative events such as death, addiction, physical and mental health diagnoses, job loss, and financial issues. P13 said of his major depression episode, "I haven't shared anything about it [on social media]... I was not talking about it with anyone." Similarly, P16 stated, "Grandparents passing away was another life event, but that's not something I really shared... because that's a really personal event that I wanted to keep to myself." $\mathrm{P} 9$ said, "I did not share... personal matters like... working with siblings that have been going through addiction." Participants' experiences show that positivity bias on social media has become a tacit rule for many, which influenced the sharing behaviors of participants and their networks, validating findings from prior work $[1,74]$. Yet people clearly do not avoid posting negative content to be disingenuous - rather, they adhere to social media norms by keeping personal and stigmatized experiences private.

In addition to positive self-presentation, participants deliberately curated their social media posts to leave desirable impressions to different audiences. Again, participants' behaviors contradict their definitions of online authenticity, which many said involved curating online content as little as possible. Such patterns are particularly prevalent on certain platforms. P11 said of Instagram, "It seems everyone wants to be professional there. You never see anything negative." Due to such norms, P11 stopped using social media when going through a breakup so she "would not be tempted to talk about it or do anything she would regret." P23 used Instagram to promote his business and put effort into following its social norms: "Instagram, that's a little bit younger audience... So you need to get something that's going to catch their attention, some flashy, some cool...Try not to look old, basically." Online spaces oriented towards professionals also witness heavy impression management. P10 described presenting herself carefully to an online community in her field: "There's more pressure to be a certain person and have a certain image....You're presenting yourself to sponsors... So it's always best to present yourself in a pretty good light..." People often curate their online presence differently on professional social media sites vs. family-or-friends-oriented sites. P13 compared LinkedIn and Facebook:

On LinkedIn, I'm very concerned about how people view me, and I really want people to look at my profile and to click all the links... Whereas on Facebook... I express myself emotionally. So... certainly I feel like I have two sides to me, if you think about the different networks online. 
These quotes demonstrate how impression management [29] can be at odds with consistency across social media sites, and how people manage context collapse by presenting themselves and disclosing information differently in different online spaces [23]. As P11 put it, people "put on different personas" across different platforms. Participants carefully curate content to conform to sites' social norms and the audiences present on each.

Some said they had shared negative life events in the past, but did not receive expected reactions and chose not to share such content again. Without receiving social support, several described sharing negative content as "pointless." P11 said,

I did try once to ask for support on social media in a personal way... I got very, very superficial things like, 'Hey, we should talk,' and then I tried to talk to them and they'd never actually follow up. Whenever I've seen people post about life crisis on social media, it's very superficial...

P28 encountered similar responses after posting on social media about becoming homeless and experiencing financial difficulties:

The reaction was not where I expected it to be. So I didn't really get any help or any info or anything. I think a lot of times people... I call it a performance... They say the thing that they feel like they're supposed to say in certain situations.

These quotes demonstrate desire for social support but highlight how social media audiences sometimes fail to deliver. Previous research has shown ways people have found support online, but often in anonymous spaces separate from one's typical online networks [3]. A lack of social support can discourage disclosing future negative content, further reducing negative self-presentation online.

This section describes how people selectively disclosed life transitions and how their online sharing behaviors contradicted common perceptions of online authenticity. P8, who chose not to share about her pregnancy due to fear that the pregnancy might end badly, drew attention to the contradiction between her beliefs about authenticity and her actual actions: "I know this [not sharing the potentially negative event] is somewhat contradictory with what I shared earlier and I don't necessarily know how to reconcile it." It is unclear how many people are aware of the contradiction P8 mentioned, but we show that the contradiction is quite prevalent. Multiple factors - widely perceived positivity bias, impression management, context collapse, and unsupportive responses when sharing negative events - lead to a discrepancy between online behavior and a desire to be honest and consistent.

\section{DISCUSSION}

We have described how online authenticity can present a paradox for social media users who desire to present themselves authentically, yet face challenges because authenticity requires high levels of self-disclosure. Business researchers introduced the authenticity paradox to describe how effective leadership often involves departing from rigid notions of authenticity, as thinking and acting in accordance with a rigid authentic "true self" can limit growing, changing, and fulfilling new roles and challenges [34, 43]. We extend this idea by explaining how authenticity can also be paradoxical for the general public when presenting identity in online spaces, a concept we call the online authenticity paradox.

Our results indicate how context collapse complicates online authenticity and self-disclosure on social media. "Online" is not one space - it involves multiple sites and communities, with different audiences, norms, and contexts, and each influences people's choices to disclose personal information about their identities and life experiences [2,55]. When, as the literature has documented, context collapse requires multiplicity and separation in online presentation [23, 55, 68, 73], and, 
as we have shown, people consider online authenticity as requiring high levels of self-disclosure with broad social media audiences, it can be difficult for people to reconcile their need to manage context collapse with their desire to be authentic. The online authenticity paradox extends this prior work by providing a new way to describe the paradoxical way context collapse influences online identity presentation. As participants in our study noted, being authentic requires sharing personal experiences online, even when such disclosures involve negative experiences or self-presentation Yet in practice, doing so can be challenging. The online authenticity paradox describes how the proclivity toward positive self-presentations complicates transparent sharing [59]. People who do not conform to site expectations may encounter negative reactions, which further reinforces people's tendency to post positive content and to segment content across platforms [58].

Our findings substantiate prior research showing that social media favors positive presentation $[1,72,76]$ and that users behave differently depending on audiences and platforms $[20,35,55,62,68]$. Yet prior work has not explicitly examined the complex relationships between how people view online authenticity and their self-presentation and self-disclosure decisions, and prior researchers did not ask participants directly about how they felt about online authenticity, and the extent to which they considered it achievable. We expand on previous work by reporting on how participants articulated their relationship to online authenticity around self-disclosure of life transitions, and whether they considered it achievable, which adds an additional dimension to how social computing researchers understand people's experiences with self-presentation in online spaces. We show how sharing selectively is common yet often considered inauthentic. These results provide new insights by demonstrating how, paradoxically, people aspire to achieve online authenticity, yet find that they can do so only at a cost (i.e., disclosure).

We call this the online authenticity paradox:

(1) Many consider authenticity an important construct to uphold in their personal lives and online presentations.

(2) Many believe that authenticity requires sharing both positive and negative experiences with broad networks online.

(3) Thus, though they do not always recognize it, online authenticity may be unachievable for many, particularly in relation to difficult or stigmatized events (due to factors including positivity bias, impression management, and context collapse).

The online authenticity paradox does not necessarily mean that every person wants to present themselves consistently across all online and offline contexts, and in reality most people find ways to live somewhat authentically online by using multiple channels like private messages, multiple accounts, and separate online networks [4, 79, 80]. However, our results (section 4.1) highlight difficulties that arise when considering the high value that many people place on authenticity alongside common conceptions of authenticity as requiring consistency across platforms and sharing both positive and negative content online. Taken together, simultaneous demands for and barriers to online authenticity make it so that common online practices like identity multiplicity and separation are often considered inauthentic [38]. In fact, Mark Zuckerberg himself has famously posited online multiplicity as inauthentic [45], and this value permeates Facebook [15, 38], the social media site participants used most frequently - which may have influenced participants' conceptions of online authenticity as being both valuable and achievable, despite their own and others' difficulty achieving it.

At the heart of the online authenticity paradox is the important concern that many people want to behave in ways they consider authentic online, yet feel they cannot. The same factors that create barriers to online authenticity - positivity bias, impression management, and context collapse - also inhibit people's ability to share content about life transitions online, especially 
related to stigmatized identities. For example (as described in our results section 4.3), P11 and P28 both experienced a lack of support from their online networks after posting about difficult negative experiences on social media, which discouraged them from posting similar content in the future. Instead, research has shown that people turn to small groups of close connections or private channels [37, 41, 80], anonymous online sharing [4, 46], and multiple or separate online identities $[20,35,79]$ to achieve online sharing that feels authentic to them. In what follows, we describe future directions for design and research that can help to resolve some of the tensions the online authenticity paradox highlights.

\subsection{Moving Forward}

5.1.1 Implications for Design. The online authenticity paradox is important to consider in designing online spaces and considering how online communities may be constructed and managed going forward. Despite the difficulties people face with online sharing, we must acknowledge that humans desire to share their feelings and experiences in ways that feel honest and consistent. Online platforms can use the online authenticity paradox to provide better user experiences, such as by facilitating connection between people with similar experiences, allowing anonymous sharing, and enabling multiple accounts and self-presentations. While online authenticity will remain elusive, such affordances and features may bring social media users closer to genuine self-presentation along with network and content separation. Each of these design implications have been suggested in previous work (e.g., [47, 52, 67, 80]), yet expounding the online authenticity paradox provides new insight on how they may be implemented. We describe how each of these design implications helps to either resolve or provide alternative solutions to the challenges the online authenticity paradox highlights, and may enable people to uphold their conception of authenticity as an important personal value. We use the numbers (1)-(3), to refer to the three parts of the online authenticity paradox, as described above.

Connecting people with similar experiences and identities. Platforms could better enable users to easily find people facing similar situations (in the past or the present), both within and outside their typical online networks (drawing from design recommendations for peer connection and support described in previous work [3, 24, 25, 47, 52, 66, 67]). Features that visualize the prevalence of negative or stigmatized life events may alleviate stress and assure users that they are not the only one in the situation [2]. Such features may enable people to receive social support from people with similar experiences. Facilitating connections with people who have similar experiences that they may want to share but have difficulty doing so due to self-presentation concerns will provide an audience for whom a person can present in a way that will likely feel more authentic to them than attempting to share such information with broad audiences or existing close ties. In this way, connections with similar others enable people to uphold their belief in authenticity's importance (1), provide an alternative solution for the challenge of sharing negative experiences with broad online networks (2), and provide one way to resolve barriers to online authenticity (3).

Anonymous and pseudonymous sharing. Rho et al. [60] described how a configuration of anonymous posts and identifiable responses on Facebook enabled a way for low-income college students to receive social support from their peers without the stigma associated with publicly disclosing one's socioeconomic status. While some platforms already do support anonymous and pseudonymous sharing $[4,46]$, identified social media platforms such as Facebook could integrate functionality for anonymous posting across a wider range of difficult and stigmatized identities and experiences, such as the life transitions contexts participants in our study described. Anonymous or pseudonymous posting would enable users to disclose negative experiences and receive social support in response without being identified, which could potentially educate a wider audience and reduce perceived stigma while protecting users' privacy, particularly on social media sites 
where identified accounts are currently expected. Depending on whether the poster considers anonymous posting to be an authentic form of online self-expression, increased affordances for anonymity and pseudonymity would either uphold or provide an alternative solution for people's belief in authenticity's importance (1). Anonymity and pseudonymity would enable sharing negative experiences with broad online networks (2), and provide an alternative solution to the challenge of online authenticity's potential unachievability (3).

Multiple accounts and self-presentations on one platform. Some social media sites (e.g., Instagram, Twitter) allow users to have multiple accounts on one platform and easily switch between them, giving users more control over self-presentation and reducing the need to switch among multiple social media platforms. Other social media sites could also integrate such capabilities, with special attention to enabling seamless transitions between different accounts. Social computing researchers have discussed the ways that, for instance, throwaway accounts on Reddit enable people to create separate sociotechnical identities for content that they do not feel comfortable associating with their primary online identity $[4,19,46]$. Even on an identified site like Facebook, where multiple accounts are not allowed, users nevertheless use multiple accounts to present multiple identities, each of which they may consider authentic [49]. Additionally, platforms could experiment with ways to alter one's self-presentation and audience for particular content without having to fully switch accounts. While some people would consider posting via multiple accounts authentic, others may not, thus multiple accounts would either uphold or provide an alternative solution for people's desire to behave authentically (1), and either resolves or provides an alternative solution to the challenge of online authenticity's potential unachievability (3). Additionally, multiple accounts provide an alternative solution for the challenge of sharing negative experiences with broad online networks (2).

These features would enable people to more easily manage audiences and curate their online self-presentation(s), and potentially find online spaces where they can behave in ways that make them feel more authentic. Thus, the design implications we suggest may reduce the tensions the online authenticity paradox describes, and help users navigate these tensions. However, each of these design suggestions may also involve negative implications. For instance, overwhelming focus on connections based on similarities may increase online echo chambers and reduce meaningful connections between people across differences, and online anonymity, despite its benefits for sharing stigmatized content $[4,60]$, is also known to decrease inhibitions and enable harmful online activity [69]. Thus, designers should consider and mitigate each of these potential negative aspects before implementing our design suggestions.

5.1.2 Implications for Research. It may be the case that we - researchers, the popular press, participants in our study, and social media users broadly - fail to capture authenticity's complexity because we have become accustomed to using casual rhetoric to describe a complex and slippery concept. Social media users have a long history of navigating platforms' sociotechnical features to present identity and disclose personal information to the extent they feel comfortable with, even when confronted with large, diverse, and incongruent online audiences [73]. Though our results show that people strive to achieve authenticity in their online self-presentation, and largely view such ideals as achievable, in practice people tend to employ much more complexity in their social media sharing behaviors. Difficulties arise when a multifaceted concept - authenticity - poses as a simple value. While it is unlikely that the word authenticity's use in the English language and in Western cultures will change substantially to embrace more complexity, the online authenticity paradox highlights that as researchers, we can and should be more precise when employing the concept of authenticity. 
This research provokes questions about how to think about and measure online authenticity. In qualitative research, how can we best study online authenticity as a value that people strive for yet generally cannot achieve? In quantitative research, how can instruments be constructed that measure authenticity in more holistic and complex ways? Is this even possible? We have described how authenticity is highly subjective and socially constructed $[32,56]$, and many social constructionists believe there is no such thing as a "real" or "true" self to remain authentic to $[12,26,27]$. Thus, common research practices such as referring to authenticity as "accurate" selfpresentation [48, 70], measuring authenticity as a discrepancy between "ideal" and "actual" self [59], or using questions that invoke "the real me" [48, 78], in some ways rebuke research participants for being unable to achieve something potentially unachievable. Based on our results, such research tools could be better constructed with a greater understanding of how people perceive online authenticity and people's complex online sharing behaviors. For example, rather than asking about authenticity directly, researchers could ask more specifically about particular audiences or sharing behaviors in different social contexts. Such an approach acknowledges that participants may not feel able to consistently present one "true" or "real" self in all settings, but rather makes self-presentational decisions depending on context.

Given that for many people online authenticity is only achievable at great personal cost (if it is achievable at all), and that authenticity is more achievable for people with higher well-being $[59,78]$ and fewer negative experiences, it is worth considering whether authenticity should be used as a research construct. Doing so may uphold the idea that identity singularity is desirable and achievable, and perpetuates social stigma against those with complex identities and who have experienced negative events that they are uncomfortable sharing with broad social media audiences. Research instruments that attempt to measure online authenticity must account for its subjective and potentially unachievable nature; creating such tools would be challenging, and perhaps of limited use given sharing behaviors' complexity. Instead, it may be time to retire online authenticity in favor of an understanding that people share content with different online audiences according to personal comfort with disclosure, site norms, and network characteristics. That is, we can apply Gergen's [26] concept of pastiche personality to online settings and reduce feelings of inauthenticity if we encourage wide acceptance that online authenticity may not be achievable after all. To the extent that a "true self" exists online, it exists multiply and in fragments across different online spaces and networks.

\subsection{Limitations and Future Work}

We first acknowledge potential sampling biases. We only recruited people who have shared major life events on social media; however, the experience of people who have experienced but not shared about life transitions online should be examined in future work. Next, participants in our study experienced a limited set of life transitions (detailed in Table 2), and thus results may not hold for other types of life events not included in our sample - an area for future work. In addition, despite diverse demographics, participates in our study all lived in North America. We acknowledge people's life transitions and social media behavior varies in other cultures; future research should consider and address them. Moreover, future researchers can expand on our work by examining online authenticity in the context of additional or specific types of life transitions, as our study has revealed the complexity and diversity of major life events.

\section{CONCLUSION}

We have examined how people think about online authenticity, uncovering contradictions between what they consider "authentic" and their own self-presentations. According to participants in our study, online authenticity requires presenting identity consistently and sharing both positive 
and negative content online - neither of which participants reported achieving. When selective self-presentation and segmenting online participation across multiple social media networks is considered inauthentic, this may be harmful for those with stigmatized identities or difficult life experiences. These results demonstrate how the concept of authenticity sometimes falls short when accounting for the faceted nature of human lives.

\section{ACKNOWLEDGMENTS}

Thanks to the participants in this study for sharing their experiences with us, and to the anonymous reviewers for their helpful feedback.

\section{REFERENCES}

[1] Nazanin Andalibi. 2020. Disclosure, Privacy, and Stigma on Social Media: Examining Non-Disclosure of Distressing Experiences. ACM Transactions on Computer-Human Interaction 27, 3 (May 2020), 18:1-18:43. https://doi.org/10.1145/ 3386600

[2] Nazanin Andalibi and Andrea Forte. 2018. Announcing Pregnancy Loss on Facebook: A Decision-Making Framework for Stigmatized Disclosures on Identified Social Network Sites. In Proceedings of the 2018 CHI Conference on Human Factors in Computing Systems (CHI '18). ACM, New York, NY, USA, 158:1-158:14. https://doi.org/10.1145/3173574.3173732

[3] Nazanin Andalibi, Oliver L. Haimson, Munmun De Choudhury, and Andrea Forte. 2018. Social Support, Reciprocity, and Anonymity in Responses to Sexual Abuse Disclosures on Social Media. ACM Transactions on Computer-Human Interaction 25, 5 (Oct. 2018), 1-35. https://doi.org/10.1145/3234942

[4] Nazanin Andalibi, Oliver L. Haimson, Munmun De Choudhury, and Andrea Forte. 2016. Understanding Social Media Disclosures of Sexual Abuse Through the Lenses of Support Seeking and Anonymity. In Proceedings of the 2016 CHI Conference on Human Factors in Computing Systems (CHI '16). ACM, New York, NY, USA, 3906-3918. https://doi.org/10.1145/2858036.2858096

[5] Mary Anderson Anderson, Jane Goodman, and Nancy K. Schlossberg. 2011. Counseling Adults in Transition, Fourth Edition: Linking Schlossberg's Theory With Practice in a Diverse World. Springer Publishing Company. Google-Books-ID: 0HMao41pDB4C.

[6] Sarah Banet-Weiser. 2012. Authentic ${ }^{T M}$ : The Politics of Ambivalence in a Brand Culture. NYU Press.

[7] John A. Bargh, Katelyn Y. A. McKenna, and Grainne M. Fitzsimons. 2002. Can You See the Real Me? Activation and Expression of the "True Self" on the Internet. Journal of Social Issues 58, 1 (Jan. 2002), 33-48. https://doi.org/10.1111/ 1540-4560.00247

[8] George A Bonanno and Stacey Kaltman. 2001. The varieties of grief experience. Clinical Psychology Review 21, 5 (July 2001), 705-734. https://doi.org/10.1016/S0272-7358(00)00062-3

[9] danah boyd. 2007. None of this is real: Identity and participation in Friendster. In Structures of Participation in Digital Culture. Social Science Research Council.

[10] Jed R. Brubaker, Gillian R. Hayes, and Paul Dourish. 2013. Beyond the Grave: Facebook as a Site for the Expansion of Death and Mourning. The Information Society 29, 3 (May 2013), 152-163. https://doi.org/10.1080/01972243.2013.777300

[11] Moira Burke and Robert Kraut. 2013. Using Facebook After Losing a Job: Differential Benefits of Strong and Weak Ties. In Proceedings of the 2013 Conference on Computer Supported Cooperative Work (CSCW'13). ACM, New York, NY, USA, 1419-1430. https://doi.org/10.1145/2441776.2441936

[12] Judith Butler. 1999. Gender Trouble. Routledge, New York.

[13] Stevie Chancellor, George Nitzburg, Andrea Hu, Francisco Zampieri, and Munmun De Choudhury. 2019. Discovering Alternative Treatments for Opioid Use Recovery Using Social Media. In Proceedings of the 2019 CHI Conference on Human Factors in Computing Systems (CHI '19). ACM, Glasgow, Scotland Uk, 1-15. https://doi.org/10.1145/3290605.3300354

[14] Stephenie R. Chaudoir and Jeffrey D. Fisher. 2010. The disclosure processes model: Understanding disclosure decision making and postdisclosure outcomes among people living with a concealable stigmatized identity. Psychological Bulletin 136, 2 (2010), 236-256. https://doi.org/10.1037/a0018193

[15] Alexander Cho. 2017. Default publicness: Queer youth of color, social media, and being outed by the machine. New Media \& Society 20, 9 (Dec. 2017), 3183-3200. https://doi.org/10.1177/1461444817744784

[16] Angela M. Cirucci. 2015. Redefining privacy and anonymity through social networking affordances. First Monday 20, 7 (June 2015). https://doi.org/10.5210/fm.v20i7.5465

[17] Juliet Corbin and Anselm Strauss. 2008. Basics of qualitative research: Techniques and procedures for developing grounded theory. Sage.

[18] Stuart Cunningham and David Craig. 2017. Being 'really real' on YouTube: authenticity, community and brand culture in social media entertainment. Media International Australia 164, 1 (Aug. 2017), 71-81. https://doi.org/10.1177/ 
1329878X17709098 Publisher: SAGE Publications Ltd.

[19] Munmun De Choudhury and Sushovan De. 2014. Mental health discourse on reddit: Self-disclosure, social support, and anonymity. Proc. ICWSM, AAAI (2014). http://www.munmund.net/pubs/icwsm_14_1.pdf

[20] Michael Ann Devito, Ashley Marie Walker, and Jeremy Birnholtz. 2018. "Too Gay for Facebook": Presenting LGBTQ+ Identity Throughout the Personal Social Media Ecosystem. Proceedings of the ACM Human Computer Interaction (PACM) 2, CSCW (2018), 23.

[21] Judith S. Donath. 1998. Identity and Deception in the Virtual Community. In Communities in Cyberspace. Routledge.

[22] Brooke Erin Duffy and Ngai Keung Chan. 2019. "You never really know who's looking": Imagined surveillance across social media platforms. New Media \& Society 21, 1 (Jan. 2019), 119-138. https://doi.org/10.1177/1461444818791318

[23] Stefanie Duguay. 2014. "He has a way gayer Facebook than I do": Investigating sexual identity disclosure and context collapse on a social networking site. New Media \& Society (Sept. 2014), 1461444814549930. https://doi.org/10.1177/ 1461444814549930

[24] Jordan Eschler and Wanda Pratt. 2017. "I'm so glad I met you": Designing Dynamic Collaborative Support for Young Adult Cancer Survivors. In Proceedings of the 2017 ACM Conference on Computer Supported Cooperative Work and Social Computing (CSCW'17). Association for Computing Machinery, New York, NY, USA, 1763-1774. https: //doi.org/10.1145/2998181.2998326

[25] Jeana Frost and Michael Massagli. 2008. Social Uses of Personal Health Information Within PatientsLikeMe, an Online Patient Community: What Can Happen When Patients Have Access to One Another's Data. fournal of Medical Internet Research 10, 3 (May 2008), e1053. https://doi.org/10.2196/jmir.1053 Company: Journal of Medical Internet Research Distributor: Journal of Medical Internet Research Institution: Journal of Medical Internet Research Label: Journal of Medical Internet Research Publisher: JMIR Publications Inc., Toronto, Canada.

[26] Kenneth Gergen. 2000. The Saturated Self: Dilemmas Of Identity In Contemporary Life (reprint edition ed.). Basic Books, New York.

[27] Anthony Giddens. 1991. Modernity and Self-identity: Self and Society in the Late Modern Age. Stanford University Press.

[28] Omri Gillath, Amanda K. Sesko, Phillip R. Shaver, and David S. Chun. 2010. Attachment, authenticity, and honesty: Dispositional and experimentally induced security can reduce self- and other-deception. fournal of Personality and Social Psychology 98, 5 (2010), 841-855. https://doi.org/10.1037/a0019206 Place: US Publisher: American Psychological Association.

[29] Erving Goffman. 1959. Presentation of Self in Everyday Life. Doubleday Anchor Books, New York, NY.

[30] Erving Goffman. 1963. Stigma: Notes on the Management of Spoiled Identity. Simon and Schuster.

[31] Mary L. Gray. 2009. Negotiating Identities/Queering Desires: Coming Out Online and the Remediation of the ComingOut Story. Journal of Computer-Mediated Communication 14, 4 (July 2009), 1162-1189. https://doi.org/10.1111/j.10836101.2009.01485.x

[32] David Grazian. 2005. Blue Chicago: The Search for Authenticity in Urban Blues Clubs. University of Chicago Press.

[33] Rachel Grieve and Jarrah Watkinson. 2016. The Psychological Benefits of Being Authentic on Facebook. Cyberpsychology, Behavior, and Social Networking 19, 7 (July 2016), 420-425. https://doi.org/10.1089/cyber.2016.0010 Publisher: Mary Ann Liebert, Inc., publishers.

[34] Eric Guthey and Brad Jackson. 2005. CEO Portraits and the Authenticity Paradox* Fournal of Management Studies 42, 5 (2005), 1057-1082. https://doi.org/10.1111/j.1467-6486.2005.00532.x _eprint: https://onlinelibrary.wiley.com/doi/pdf/10.1111/j.1467-6486.2005.00532.x.

[35] Oliver L. Haimson. 2018. Social Media as Social Transition Machinery. Proc. ACM Hum.-Comput. Interact. 2, CSCW (Nov. 2018), 63:1-63:27. https://doi.org/10.1145/3274332

[36] Oliver L. Haimson, Nazanin Andalibi, Munmun De Choudhury, and Gillian R. Hayes. 2018. Relationship breakup disclosures and media ideologies on Facebook. New Media \& Society 20, 5 (2018). https://doi.org/10.1177/1461444817711402

[37] Oliver L. Haimson, Albert J. Carter, Shanley Corvite, Brookelyn Wheeler, Lingbo Wang, Tianxiao Liu, and Alexxus Lige. 2021. The Major Life Events Taxonomy: Social Readjustment, Social Media Information Sharing, and Online Network Separation During Times of Life Transition. Journal of the Association for Information Science and Technology (JASIST) (2021)

[38] Oliver L. Haimson and Anna Lauren Hoffmann. 2016. Constructing and enforcing "authentic" identity online: Facebook, real names, and non-normative identities. First Monday 21, 6 (June 2016). https://doi.org/10.5210/fm.v21i6.6791

[39] Erin E. Hardin and Jessica L. Lakin. 2009. The Integrated Self-Discrepancy Index: A Reliable and Valid Measure of SelfDiscrepancies. Journal of Personality Assessment 91, 3 (May 2009), 245-253. https://doi.org/10.1080/00223890902794291

[40] Alison Hearn. 2017. Verified: Self-presentation, identity management, and selfhood in the age of big data. Popular Communication 15, 2 (April 2017), 62-77. https://doi.org/10.1080/15405702.2016.1269909

[41] Jeremy Heyer, Zachary Schmitt, Lynn Dombrowski, and Svetlana Yarosh. 2020. Opportunities for Enhancing Access and Efficacy of Peer Sponsorship in Substance Use Disorder Recovery. In Proceedings of the 2020 CHI Conference on Human Factors in Computing Systems (CHI '20). Association for Computing Machinery, Honolulu, HI, USA, 1-14.

Proc. ACM Hum.-Comput. Interact., Vol. 5, No. CSCW2, Article 423. Publication date: October 2021. 
https://doi.org/10.1145/3313831.3376241

[42] Vera Hoorens. 2014. Positivity Bias. In Encyclopedia of Quality of Life and Well-Being Research, Alex C. Michalos (Ed.). Springer Netherlands, Dordrecht, 4938-4941. https://doi.org/10.1007/978-94-007-0753-5_2219

[43] Herminia Ibarra. 2015. The Authenticity Paradox: Why feeling like a fake can be a sign of growth. Harvard Business Review (Feb. 2015), 9.

[44] C. David Jenkins, Michael W. Hurst, and Robert M. Rose. 1979. Life Changes: Do People Really Remember? Archives of General Psychiatry 36, 4 (April 1979), 379-384. https://doi.org/10.1001/archpsyc.1979.01780040021001

[45] David Kirkpatrick. 2010. The Facebook Effect: The Inside Story of the Company That Is Connecting the World. Simon and Schuster.

[46] Alex Leavitt. 2015. "This is a Throwaway Account": Temporary Technical Identities and Perceptions of Anonymity in a Massive Online Community. In Proceedings of the 18th ACM Conference on Computer Supported Cooperative Work \&\#38; Social Computing (CSCW '15). ACM, New York, NY, USA, 317-327. https://doi.org/10.1145/2675133.2675175

[47] Zachary Levonian, Marco Dow, Drew Erikson, Sourojit Ghosh, Hannah Miller Hillberg, Saumik Narayanan, Loren Terveen, and Svetlana Yarosh. 2021. Patterns of Patient and Caregiver Mutual Support Connections in an Online Health Community. Proceedings of the ACM on Human-Computer Interaction 4, CSCW3 (Jan. 2021), 275:1-275:46. https://doi.org/10.1145/3434184

[48] Joon Soo Lim, John Nicholson, Sung-Un Yang, and Ho-Kyung Kim. 2015. Online authenticity, popularity, and the "Real Me" in a microblogging environment. Computers in Human Behavior 52 (Nov. 2015), 132-143. https: //doi.org/10.1016/j.chb.2015.05.037

[49] Jessa Lingel and Adam Golub. 2015. In Face on Facebook: Brooklyn's Drag Community and Sociotechnical Practices of Online Communication. Journal of Computer-Mediated Communication (June 2015), n/a-n/a. https://doi.org/10.1111/ jcc4.12125

[50] Frederick G. Lopez and Kenneth G. Rice. 2006. Preliminary development and validation of a measure of relationship authenticity. Journal of Counseling Psychology 53, 3 (2006), 362-371. https://doi.org/10.1037/0022-0167.53.3.362 Place: US Publisher: American Psychological Association.

[51] Haiwei Ma, C. Estelle Smith, Lu He, Saumik Narayanan, Robert A. Giaquinto, Roni Evans, Linda Hanson, and Svetlana Yarosh. 2017. Write for Life: Persisting in Online Health Communities Through Expressive Writing and Social Support. Proc. ACM Hum.-Comput. Interact. 1, CSCW (Dec. 2017), 73:1-73:24. https://doi.org/10.1145/3134708

[52] Haley MacLeod, Grace Bastin, Leslie S. Liu, Katie Siek, and Kay Connelly. 2017. "Be Grateful You Don't Have a Real Disease": Understanding Rare Disease Relationships. In Proceedings of the 2017 CHI Conference on Human Factors in Computing Systems (CHI '17). Association for Computing Machinery, New York, NY, USA, 1660-1673. https://doi.org/10.1145/3025453.3025796

[53] Alice E. Marwick. 2005. 'I'm a Lot More Interesting than a Friendster Profile': Identity Presentation, Authenticity and Power in Social Networking Services. SSRN Scholarly Paper ID 1884356. Social Science Research Network, Rochester, NY. http://papers.ssrn.com/abstract $=1884356$

[54] Alice E. Marwick. 2013. Online Identity. In A Companion to New Media Dynamics, John Hartley, Jean Burgess, and Axel Bruns (Eds.). Wiley-Blackwell, 355-364. http://onlinelibrary.wiley.com/doi/10.1002/9781118321607.ch23/summary

[55] Alice E. Marwick and danah boyd. 2010. I tweet honestly, I tweet passionately: Twitter users, context collapse, and the imagined audience. New Media \& Society 13, 1 (Feb. 2010), 114-133. https://doi.org/10.1177/1461444810365313

[56] Richard A. Peterson. 2005. In Search of Authenticity*. fournal of Management Studies 42, 5 (July 2005), $1083-1098$. https://doi.org/10.1111/j.1467-6486.2005.00533.x

[57] Derek L. Phillips and Kevin J. Clancy. 1972. Some Effects of "Social Desirability" in Survey Studies. Amer. F. Sociology 77, 5 (1972), 921-940. https://www.jstor.org/stable/2776929 Publisher: University of Chicago Press.

[58] Mikaela Pitcan, Alice E. Marwick, and Danah Boyd. 2018. Performing a Vanilla Self: Respectability Politics, Social Class, and the Digital World. Journal of Computer-Mediated Communication 23, 3 (May 2018), 163-179. https: //doi.org/10.1093/jcmc/zmy008 Publisher: Oxford Academic.

[59] Leonard Reinecke and Sabine Trepte. 2014. Authenticity and well-being on social network sites: A two-wave longitudinal study on the effects of online authenticity and the positivity bias in SNS communication. Computers in Human Behavior 30 (Jan. 2014), 95-102. https://doi.org/10.1016/j.chb.2013.07.030

[60] Eugenia Ha Rim Rho, Oliver L. Haimson, Nazanin Andalibi, Melissa Mazmanian, and Gillian R. Hayes. 2017. Class Confessions: Restorative Properties in Online Experiences of Socioeconomic Stigma. In Proceedings of the 2017 CHI Conference on Human Factors in Computing Systems (CHI '17). ACM, New York, NY, USA, 3377-3389. https: //doi.org/10.1145/3025453.3025921

[61] Bernard Rimé. 2009. Emotion Elicits the Social Sharing of Emotion: Theory and Empirical Review:. Emotion Review (Jan. 2009). https://doi.org/10.1177/1754073908097189 Publisher: SAGE PublicationsSage UK: London, England.

[62] Jenny Rosenberg and Nichole Egbert. 2011. Online Impression Management: Personality Traits and Concerns for Secondary Goals as Predictors of Self-Presentation Tactics on Facebook. Fournal of Computer-Mediated Communication 
17, 1 (Oct. 2011), 1-18. https://doi.org/10.1111/j.1083-6101.2011.01560.x Publisher: Oxford Academic.

[63] Sarita Schoenebeck, Nicole B. Ellison, Lindsay Blackwell, Joseph B. Bayer, and Emily B. Falk. 2016. Playful Backstalking and Serious Impression Management: How Young Adults Reflect on their Past Identities on Facebook. In Proceedings of the 19th ACM Conference on Computer-Supported Cooperative Work \& Social Computing (CSCW '16). Association for Computing Machinery, San Francisco, California, USA, 1475-1487. https://doi.org/10.1145/2818048.2819923

[64] Gwendolyn Seidman. 2014. Expressing the "True Self" on Facebook. Computers in Human Behavior 31 (Feb. 2014), 367-372. https://doi.org/10.1016/j.chb.2013.10.052

[65] Bryan Semaan, Lauren M. Britton, and Bryan Dosono. 2017. Military Masculinity and the Travails of Transitioning: Disclosure in Social Media. In Proceedings of the 2017 ACM Conference on Computer Supported Cooperative Work and Social Computing (CSCW '17). ACM, New York, NY, USA, 387-403. https://doi.org/10.1145/2998181.2998221

[66] Jane M. Simoni, Julie C. Franks, Keren Lehavot, and Samantha S. Yard. 2011. Peer interventions to promote health: conceptual considerations. The American fournal of Orthopsychiatry 81, 3 (July 2011), 351-359. https://doi.org/10. 1111/j.1939-0025.2011.01103.x

[67] C. Estelle Smith, Zachary Levonian, Haiwei Ma, Robert Giaquinto, Gemma Lein-Mcdonough, Zixuan Li, Susan O'conner-Von, and Svetlana Yarosh. 2020. "I Cannot Do All of This Alone": Exploring Instrumental and Prayer Support in Online Health Communities. ACM Transactions on Computer-Human Interaction 27, 5 (Aug. 2020), 38:1-38:41. https://doi.org/10.1145/3402855

[68] Frederic Stutzman and Woodrow Hartzog. 2012. Boundary Regulation in Social Media. In Proceedings of the ACM 2012 Conference on Computer Supported Cooperative Work (CSCW '12). ACM, New York, NY, USA, 769-778. https: //doi.org/10.1145/2145204.2145320

[69] John Suler. 2004. The Online Disinhibition Effect. CyberPsychology \& Behavior 7, 3 (June 2004), 321-326. https: //doi.org/10.1089/1094931041291295

[70] Lee Taber and Steve Whittaker. 2020. "On Finsta, I can say 'Hail Satan'": Being Authentic but Disagreeable on Instagram. In Proceedings of the 2020 CHI Conference on Human Factors in Computing Systems. ACM, Honolulu HI USA, 1-14. https://doi.org/10.1145/3313831.3376182

[71] Sabine Trepte and Leonard Reinecke. 2011. The Social Web as a Shelter for Privacy and Authentic Living. In Privacy Online, Sabine Trepte and Leonard Reinecke (Eds.). Springer Berlin Heidelberg, Berlin, Heidelberg, 61-73. http://link.springer.com/10.1007/978-3-642-21521-6_6

[72] Suvi Uski and Airi Lampinen. 2014. Social norms and self-presentation on social network sites: Profile work in action:. New Media \& Society 18, 3 (July 2014), 447-464. https://doi.org/10.1177/1461444814543164 Publisher: SAGE PublicationsSage UK: London, England.

[73] Jessica Vitak. 2012. The Impact of Context Collapse and Privacy on Social Network Site Disclosures. Fournal of Broadcasting \& Electronic Media 56, 4 (Oct. 2012), 451-470. https://doi.org/10.1080/08838151.2012.732140

[74] Jessica Vitak, Stacy Blasiola, Eden Litt, and Sameer Patil. 2015. Balancing Audience and Privacy Tensions on Social Network Sites: Strategies of Highly Engaged Users. International fournal of Communication 9 (2015), 20. http: //ijoc.org/index.php/ijoc/article/view/3208

[75] Erin A. Vogel, Jason P. Rose, Lindsay R. Roberts, and Katheryn Eckles. 2014. Social comparison, social media, and self-esteem. Psychology of Popular Media Culture 3, 4 (Oct. 2014), 206-222. https://doi.org/10.1037/ppm0000047

[76] Yi-Chia Wang, Hayley Hinsberger, and Robert E. Kraut. 2016. Does Saying This Make Me Look Good?: How Posters and Outsiders Evaluate Facebook Updates. In Proceedings of the 2016 CHI Conference on Human Factors in Computing Systems (CHI '16). ACM, New York, NY, USA, 125-129. https://doi.org/10.1145/2858036.2858502

[77] Sophie F. Waterloo, Susanne E. Baumgartner, Jochen Peter, and Patti M. Valkenburg. 2017. Norms of online expressions of emotion: Comparing Facebook, Twitter, Instagram, and WhatsApp:. New Media \& Society 20 (May 2017), 1813-1831. https://doi.org/10.1177/1461444817707349 Publisher: SAGE PublicationsSage UK: London, England.

[78] Alex M. Wood, P. Alex Linley, John Maltby, Michael Baliousis, and Stephen Joseph. 2008. The authentic personality: A theoretical and empirical conceptualization and the development of the Authenticity Scale. fournal of Counseling Psychology 55, 3 (2008), 385-399. https://doi.org/10.1037/0022-0167.55.3.385

[79] Sijia Xiao, Danaë Metaxa, Joon Sung Park, Karrie Karahalios, and Niloufar Salehi. 2020. Random, Messy, Funny, Raw: Finstas as Intimate Reconfigurations of Social Media. In Proceedings of the 2020 CHI Conference on Human Factors in Computing Systems (CHI '20). Association for Computing Machinery, Honolulu, HI, USA, 1-13. https: //doi.org/10.1145/3313831.3376424

[80] Diyi Yang, Zheng Yao, Joseph Seering, and Robert Kraut. 2019. The Channel Matters: Self-disclosure, Reciprocity and Social Support in Online Cancer Support Groups. In Proceedings of the 2019 CHI Conference on Human Factors in Computing Systems (CHI '19). Association for Computing Machinery, New York, NY, USA, 1-15. https://doi.org/10. $1145 / 3290605.3300261$

Received January 2021; revised April 2021; accepted July 2021

Proc. ACM Hum.-Comput. Interact., Vol. 5, No. CSCW2, Article 423. Publication date: October 2021. 